\title{
Femtosecond digital lensless holographic microscopy to image biological samples
}

\author{
Omel Mendoza-Yero, ${ }^{1}$ Alejandro Calabuig, ${ }^{1}$ Enrique Tajahuerce, ${ }^{1}$ Jesús Lancis, ${ }^{1}$ Pedro Andrés, ${ }^{2}$ \\ and Jorge Garcia-Sucerquia ${ }^{2,3, *}$ \\ ${ }^{1}$ Institut de Noves Tecnologies de la Imatge (INIT), Universitat Jaume I, 12071 Castelló, Spain \\ ${ }^{2}$ Departamento de Óptica, Universitat de València, 46100 Burjassot, Spain \\ ${ }^{3}$ Universidad Nacional de Colombia Sede Medellin, School of Physics, A.A: 3840 Medellin, 050034 Colombia \\ *Corresponding author: jigarcia@unal.edu.co
}

Received April 29, 2013; revised July 3, 2013; accepted July 15, 2013; posted July 16, 2013 (Doc. ID 189728); published August 19, 2013

\begin{abstract}
The use of femtosecond laser radiation in digital lensless holographic microscopy (DLHM) to image biological samples is presented. A mode-locked Ti:Sa laser that emits ultrashort pulses of 12 fs intensity FWHM, with $800 \mathrm{~nm}$ mean wavelength, at $75 \mathrm{MHz}$ repetition rate is used as a light source. For comparison purposes, the light from a lightemitting diode is also used. A section of the head of a drosophila melanogaster fly is studied with both light sources. The experimental results show very different effects of the pinhole size on the spatial resolution with DLHM. Unaware phenomena on the field of the DLHM are analyzed. (c) 2013 Optical Society of America

OCIS codes: (090.1995) Digital holography; (110.0180) Microscopy; (030.4280) Noise in imaging systems. http://dx.doi.org/10.1364/OL.38.003205
\end{abstract}

The coherence properties of light determine the performance of digital holographic microscopy (DHM). While two-arm DHM needs both temporal and spatial coherence to operate properly, the single-arm DHM relaxes the requirements over the former. For both architectures, continuous wave (CW) lasers have been the preferred light sources because they simplify the experimental setups. New radiation sources are now utilized in DHM to optimize its performance $[1,2]$ and/or to explore new applications []ㅡ. The utilization of femtosecond laser radiation has been proposed recently for both DHM architectures $[4,5]$. For the two-arm DHM, the limited coherence time of this source imposes experimental configurations with very short optical path differences and compensating devices to extend the field of interference $[\underline{4}, \underline{6}]$. Because the single-arm DHM relaxes the conditions over the coherence time, it simplifies the utilization of femtosecond lasers, as was presented by Brunel et al. [5]. In that work, the authors utilized 20 fs plane wave laser radiation to image with a lensless microscope a circular thin film of indium tin oxide $260 \mu \mathrm{m}$ in diameter. Beyond this type of application, the use of femtosecond laser radiation in DHM may be useful, for instance, to study the spectral response of complex internal structures in biological samples. For these applications, lensless microscopes are the preferred imaging tool because they preserve the temporal pulse duration and do not introduce chromatic aberrations. However, the imaging approach must offer larger magnification and resolution power than that provided by plane wave lensless holographic microscopes [5]. A microscopy architecture that uses no lenses and provides micrometer resolution operating at multiple wavelengths is digital lensless holographic microscopy (DLHM) $[7,8]$. DLHM can offer the features needed to image biological specimens with femtosecond laser radiation. In this Letter, the use of femtosecond laser radiation in DLHM and its application to image biological samples is presented. The variation of the pinhole size shows new and unaware effects on the performance of DLHM that are compared with the previously known.
In DLHM, the sample is illuminated by a spherical wavefront with a wavelength $\lambda$. A digital screen records the amplitude superposition of the portion of the spherical wavefront that is scattered by the specimen, $U_{\text {scat}}$, with the portion that travels from the point source to the screen with no distortion, $U_{\text {ref }}$. The former is called the object wave and the latter is the reference wave. The intensity recorded by the digital screen is transferred to a $\mathrm{PC}$ for further processing, which includes the retrieving of $U_{\text {scat }}$. A DLHM setup is illustrated in Fig. 1 . For the light sources in this study, we utilize a LED and a mode-locked Ti:Sa laser with 12 fs intensity FWHM and $800 \mathrm{~nm}$ mean wavelength at $75 \mathrm{MHz}$ repetition rate and energy per pulse of $4 \mathrm{~nJ}$. For all types of illumination sources, perhaps the most important challenge in order for DLHM to work is the production of the point source. Usually this point source is produced by focusing down light onto a pinhole with diameter $d_{p} \approx \lambda$; namely, the light is shrunk into an area of the order of $\pi \lambda^{2} / 4$. For instance, for a typical $\mathrm{CW} \mathrm{He}-\mathrm{Ne}$ laser of $10 \mathrm{~mW}$, the power per unit area over the surface of the pinhole is of the order of

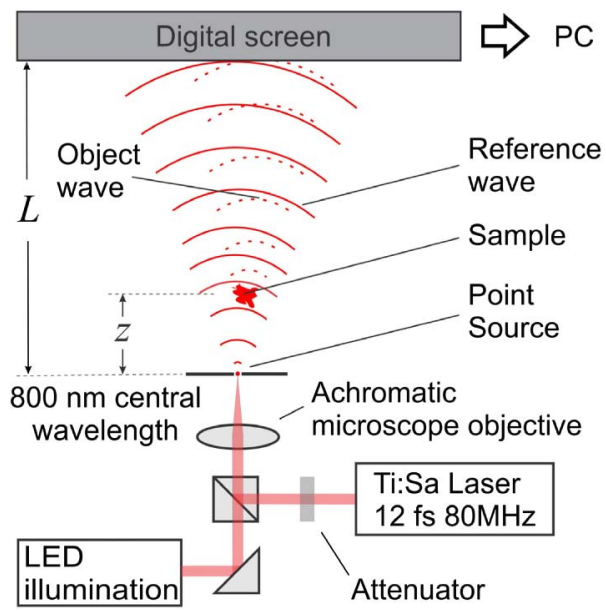

Fig. 1. Schematic setup for DLHM using both Ti:Sa laser and LED illumination. 
$0.7 \mathrm{MW} / \mathrm{cm}^{2}$. This figure is enough below the damage threshold of the commercial pinholes, which is of the order of $70 \mathrm{TW} / \mathrm{cm}^{2}$. In our application, the peak power over the surface of the pinhole after focusing the ultrashort pulse is of the order of $2.5 \mathrm{TW} / \mathrm{cm}^{2}$. This value makes advisable some reduction of the power of the light for safety reasons while enough energy is kept for recording the hologram. Hence, the peak power is reduced to about $1.1 \mathrm{TW} / \mathrm{cm}^{2}$ by decreasing the pulse energy with neutral filters.

For comparison of the noise reduction achieved with the use of LEDs $[1,2]$ and that with the fs laser in lensless holographic setups [5], we add to our setup a superbright $655 \pm 20 \mathrm{~nm}$ LED manufactured by Luxeon. For the fs DLHM as well as for the LED-DLHM, pinholes with 5 and $1 \mu \mathrm{m}$ diameters are utilized. While the $5 \mu \mathrm{m}$ pinhole is utilized for enhancing the light throughput, the $1 \mu \mathrm{m}$ pinhole optimizes the spatial resolution. For both sources, the light from the optical source is focused down onto the pinhole by using an M-40X microscope objective (manufactured by Newport). The microscope objective has a numerical aperture of 0.65 , working distance of $0.6 \mathrm{~mm}$, and effective focal length $F=4.5 \mathrm{~mm}$. The smallest (largest) spot diameter over the plane of the pinhole in our experiment is $1.2(2) \mu \mathrm{m}$. The holograms are recorded on a CCD camera (manufactured by Balser) with $1024 \times 1024$ square pixels of $6 \mu \mathrm{m}$ side. The camera (sample) is located at a distance $17(5) \mathrm{mm}$ from the pinhole.

A section of the head of a drosophila melanogaster fly is utilized as sample to study. For the fs-DLHM, the $800 \mathrm{~nm}$ mean wavelength $\lambda_{0}$ of the ultrashort pulse has been chosen as the reconstruction wavelength. The reconstructed holograms from this complex biological specimen for both the fs-DLHM and LED-DLHM are shown in Fig. 2. For all the panels, no coherence noise
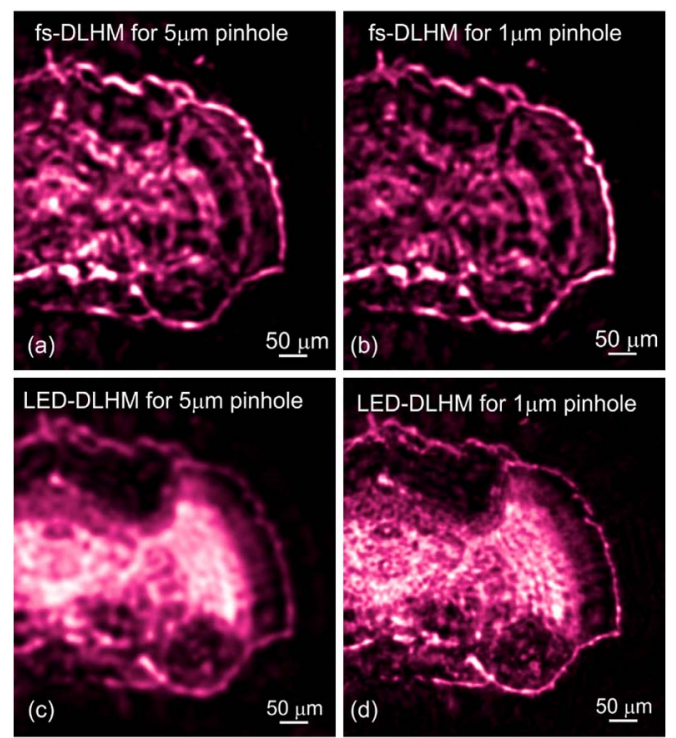

Fig. 2. Hologram reconstructions for the fs-DLHM and LEDDLHM. Panels (a) and (b) show the reconstructions for the fs-DLHM operating with pinholes of 5 and $1 \mu \mathrm{m}$ in diameter, respectively. Panels (c) and (d) are the reconstructed holograms for the LED-DLHM using pinholes with diameters of 5 and $1 \mu \mathrm{m}$, in that order. is visible, as was expected from previous works $[1,2,5]$. As the panels of the top row are compared with those in the bottom row, one can conclude that the reduction of the coherence noise that is achieved with both sources is comparable; however, there is an important difference in the spatial resolution of the reconstructed holograms. It has been previously reported that in DLHM, the achievable spatial resolution is improved as the pinhole size is reduced, essentially because the spatial coherence of the illuminating source over the plane of the sample is enhanced [1]. From Figs. 2(c) and 2(d) this premise is validated, but for Figs. $2(\mathrm{a})$ and $2(\mathrm{~b})$, this is no longer true; hence, a further analysis is needed.

To understand why the reconstructed images for the fs-DLHM are blurred, one can regard the broadband illuminating wave as being composed by a bundle of wavelengths. Thus, the recorded in-line hologram is given by the weighted incoherent superposition of multiple monochromatic in-line holograms, each one of the form

$$
\begin{aligned}
I^{\prime}(\mathbf{r}, \lambda)= & \left|U_{\text {scat }}(\mathbf{r}, \lambda)\right|^{2}+\left|U_{\text {ref }}(\mathbf{r}, \lambda)\right|^{2} \\
& +\left[U_{\text {scat }}(\mathbf{r}, \lambda) U_{\text {ref }}^{*}(\mathbf{r}, \lambda)+U_{\text {scat }}^{*}(\mathbf{r}, \lambda) U_{\text {ref }}(\mathbf{r}, \lambda)\right] .
\end{aligned}
$$

In Eq. (1), $\left|U_{\text {scat }}(\mathbf{r}, \lambda)\right|^{2}$ is negligible because the samples utilized in DLHM lightly scatter the impinging wavefront. The terms in the square brackets are the so-called twin images [7]. As spherical illumination is utilized, the presence of the twin images does not introduce any nuisance on the reconstructed image, as has been extensively studied in previous works [2,7].

The spectral power $\rho(\lambda)$ is the corresponding weighting factor such that the recorded in-line hologram in the fs-DLHM is given by

$$
I(\mathbf{r})=\int_{\lambda_{0}-\Delta \lambda} 2^{\lambda_{0}+\Delta} \lambda 2 \rho(\lambda) I^{\prime}(\mathbf{r}, \lambda) \mathrm{d} \lambda,
$$

with $\lambda_{0}$ the mean wavelength and $\Delta \lambda$ the spectral width of the illuminating source. To eliminate possible intensity inhomogeneities, the intensity recorded by the digital screen with no sample present is pixelwise subtracted from the in-line hologram to produce

$$
\tilde{I}(\mathbf{r})=\int_{\lambda_{0}-\Delta N} 2^{\lambda_{0}+\Delta} \lambda 2 \rho(\lambda)\left[I^{\prime}(\mathbf{r}, \lambda)-\left|U_{\text {ref }}(\mathbf{r}, \lambda)\right|^{2}\right] \mathrm{d} \lambda .
$$

Because the integration time of the digital screen is much longer than the temporal pulse width, the contrast hologram can be correctly expressed by the intensity superposition given in Eq. (3) [9]. The complex amplitude wave field scattered by the sample $U_{\text {scat }}$ can be retrieved by computing the diffraction that a reference wave $U_{\text {ref }}$ undergoes as it illuminates the contrast hologram $\tilde{I}(\mathbf{r})$ [7] .

As the contrast hologram can be represented by Eq. (3), the information about the individual wavelengths is merged. For that reason, the utilized reconstructing wavelength only changes the scale of the reconstructed image. Details about the numerical implementation of the diffraction process for the case of DLHM can be found elsewhere [10]. The above reasoning leads us to 


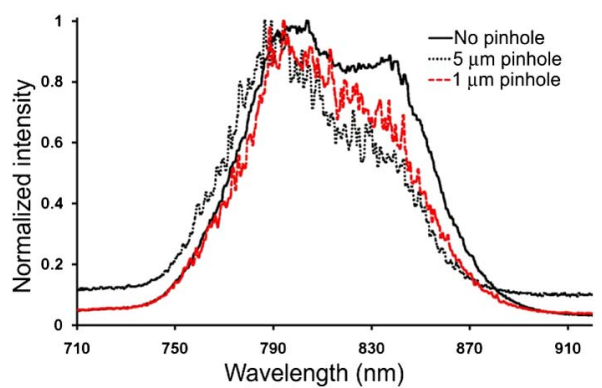

Fig. 3. Spectra of the illuminations utilized in the fs-DLHM. The solid line corresponds to illumination with no pinhole. The dotted line and the dashed line illustrate the spectrum for the 5 and $1 \mu \mathrm{m}$ pinholes, respectively.

understand $U_{\text {scat }}$ as the incoherent superposition of multiple diffracted fields from the same specimen, each one with a slightly different scale proportional to $N \lambda_{0}$; this superposition results in a blurred reconstructed image, as shown in Figs. 2(a) and 2(b).

The reason why the reconstructed images for the fsDLHM are nearly invariant with respect to the pinhole size can be supported by considering the DLHM as a wavefront-splitting interferometric microscope. Under this consideration, similar to how it happens in a Young's-like interferometer, only the spatial coherence controls the performance of the microscope. As the femtosecond laser is essentially a fully spatially coherent source, the different pinhole sizes do not modify the spatial coherence properties over the sample plane; hence, no change in the performance of the microscope is observable. For a spatially partially coherent source such as the LED, the diameter of the area that is almost coherently illuminated $D_{\text {coh }}$ in a plane located at a distance $Z$ from the pinhole is given by the Van Cittert-Zernike theorem, $D_{\text {coh }} \approx 0.32 \lambda_{0} Z / d_{p}$. The above expression indicates that the smaller the pinhole diameter, the larger the spatial coherence, and therefore the better the achievable spatial resolution of the DLHM [1,2]. This feature is verified in Figs. 2(c) and 2(d). Because the fs laser is a fully spatially coherent source, in the framework of this analysis the performance of the fs-DLHM is not modified essentially by the size of the pinhole, as shown in Figs. 2(a) and 2(b). The blurring of the reconstructed images is therefore due to the superposition of the multiple reconstructed fields with different scaling, as was mentioned above.
The above analysis is supported by measuring the spectrum of the light that illuminates the sample. The spectra that are measured for no pinhole and the pinholes of 5 and $1 \mu \mathrm{m}$ are the same, as shown in Fig. 3 . The almost identical spectra are consistent with the fact that the quality of the reconstructed images does not change with the size of the pinhole.

In summary, we have shown the use of femtosecond laser radiation in DLHM. With a suitable spectral filtering method, the information gathered with the fs-DLHM for the multiple wavelengths can be unscrambled, providing a new tool for life science imaging, as was recently shown by our group [11].

This work was funded in part by the Spanish Ministerio de Cienciae Innovación and the GeneralitatValenciana through the Consolider Programme (SAUUL CSD2007-00013), the Prometeo Excellence Programme (PROMETEO/2012/ 021), and projects FIS2010-15746, P11B2010-26, and CTQ2008-02578. J. Garcia-Sucerquia gratefully acknowledges the Visiting Scholar Fellowship from the Universidad de Valencia, Colciencias Grant No. 110205024, and UNAL Grants No. 110201003 and No. 110201004.

\section{References}

1. P. Petruck, R. Riesenberg, and R. Kowarschik, Appl. Phys. B 106, 339 (2012).

2. J. Garcia-Sucerquia, Appl. Opt. 52, A232 (2013).

3. S. O. Isikman, W. Bishara, and A. Ozcan, Appl. Opt. 50, H253 (2011).

4. S. Witte, A. Plauska, M. C. Ridder, L. van Berge, H. D. Mansvelder, and M. L. Groot, Biomed. Opt. Express 3, 2184 (2012).

5. M. Brunel, H. Shen, S. Coetmellec, D. Lebrun, and K. Ait Ameur, Appl. Phys. B 106, 583 (2012).

6. R. Martínez-Cuenca, L. Martínez-León, J. Lancis, G. Mínguez-Vega, O. Mendoza-Yero, E. Tajahuerce, P. Clemente, and P. Andrés, Opt. Express 17, 23016 (2009).

7. J. Garcia-Sucerquia, W. Xu, S. K. Jericho, P. Klages, M. H. Jericho, and H. J. Kreuzer, Appl. Opt. 45, 836 (2006).

8. J. Garcia-Sucerquia, Opt. Lett. 37, 1724 (2012).

9. M. Gu, Advanced Optical Imaging Theory, Vol. 75 of Springer Series in Optical Sciences (Springer, 2000).

10. M. H. Jericho and H. J. Kreuzer, in Coherent Light Microscopy, P. Ferraro, A. Wax, and Z. Zalevvsky, eds., Springer Series in Surface Sciences (Springer, 2011), pp. $3-30$.

11. O. Mendoza-Yero, E. Tajahuerce, J. Lancis, and J. Garcia-Sucerquia, Opt. Lett. 38, 2107 (2013). 